\title{
Frecuencia de infección por Helicobacter pylori en pacientes atendidos en el ámbito del Centro de Salud Ocaña, Ayacucho
}

\author{
Frequency of Helicobacter pylori infection in patients attended at Centro de Salud Ocaña, Ayacucho
}

John Christian Chavez Barriga ${ }^{1, a, b}$

\section{RESUMEN}

Objetivos: Determinar la frecuencia de infección por H. pylori, en pacientes atendidos en el Centro de salud de Ocaña, Ayacucho. Material y métodos: Estudio descriptivo y no experimental, transversal. El estudio se llevó a cabo en el Centro de Salud Ocaña que pertenece a la Unidad Ejecutora Salud Sur Ayacucho; en pacientes a quienes se les solicito la prueba rápida para determinación de anticuerpos séricos para $H$. pylori en el periodo comprendido entre julio a diciembre del año 2017. Resultados: La frecuencia de infección por Helicobacter pylori fue 67,3\%, la frecuencia de la infección según grupo etario fue $62,5 \%, 52,9 \%, 68,1 \%, 72,7 \%$ y 76,9\% para adolescentes, jóvenes, adultos, adultos mayores y gestantes, respectivamente, la frecuencia de infección según sexo fue 74,5\% y $64,8 \%$ para el sexo masculino y femenino, respectivamente. Conclusiones: La frecuencia encontrada es mayor que en países desarrollados, pero similar al promedio en Sudamérica, no se encuentran diferencias significativas en la distribución por sexo y grupo etario.

PALABRAS CLAVE: Helicobacter pylori, infección, prevalencia. (Fuente: DeCS BIREME).

\section{SUMMARY}

Objectives: To determine the frequency of Helicobacter pylori infection in patients attended at Centro de Salud Ocaña, Ayacucho. Methods: A cross-sectional study was performed in patients at whom a rapid H. pylori Ab was carriedout from July to December 2017. Results: The frequency of H. pylori was $67.3 \%$, the frequency of infection by age group was $62.5 \%, 52.9 \%, 68.1 \%, 72.7 \%$ y $76.9 \%$ in adolescents, young adults, elder, and pregnants, respectively. The frequency of infection by sex was $74.5 \%$ and $64.8 \%$ for males and females, respectively. Conclusions: The frequency of $\mathrm{H}$. pylori infection found in this study is higher than that reported from developed countries, but similar to that found in South American countries, no significant differences by age and sex were found.

KEYWORDS: Helicobacter pylori, infection, prevalence. (Source: DeCS BIREME).

Universidad Nacional de San Cristóbal de Huamanga, Ayacucho, Perú. 


\section{INTRODUCCIÓN}

La infección por Helicobacter pylori constituye un factor de riesgo para cáncer de estómago el cual representa la principal causa de mortalidad por cáncer en el Perú (1). Diversos estudios muestran que el 50\% de la población mundial se infecta en algún momento en la vida y que $1-3 \%$ de los infectados desarrollan cáncer gástrico en el tiempo $(2,3)$.

La colonización persistente del epitelio gástrico por este microorganismo constituye un importante factor de riesgo en la aparición de varias enfermedades gastroduodenales: gastritis crónica no autoinmune, úlcera gástrica, úlcera duodenal, cáncer gástrico y linfoma del tejido linfoide asociado a mucosa (linfoma MALT) (4). Por lo antes expuesto, este patógeno fue clasificado como carcinógeno tipo I para el hombre por la Agencia de Investigaciones del Cáncer en 1994 (5).

La ingesta de agua contaminada constituye el mecanismo más importante de infección en países en vías desarrollo. Las primeras evidencias sobre la ruta de transmisión acuática de este microorganismo a humanos fueron aportadas por estudios epidemiológicos realizados en países en vías de desarrollo como Perú, Colombia, Chile y Venezuela (6).

El consumo de agua no tratada es considerado un factor de riesgo para la infección en poblaciones $(7,8)$.

La prueba de inmunoserología rápida por la técnica de inmunocromatografía de flujo lateral la permite la detección en forma conjunta de IgM, IgG e IgA; para la muestra se utiliza una gota de sangre periférica capilar de pulpejo de dedo.

En el distrito de Ocaña en personal de salud se orienta a la prevención de enfermedades asociadas a H. pylori para desarrollar estrategias que contemplen la detección precoz y oportuna del agente infeccioso para evitar las complicaciones del mismo que repercuten en la salud individual y publica por su forma de transmisión hace necesario vigilar la frecuencia de $\mathrm{H}$. pylori en la población mediante técnicas sencillas, confiables y uso masivo basadas en el inmunodiagnostico que ofrezca un buen resultado en la detección del agente patógeno.

El objetivo general del estudio fue determinar la frecuencia de la infección por Helicobacter pylori en pacientes atendidos en el ámbito del Centro de Salud Ocaña.

\section{MATERIAL Y MÉTODOS}

Estudio descriptivo, no experimental, transversal, realizado en el Centro de Salud Ocaña, Ocaña, provincia Lucanas, Departamento de Ayacucho. La muestra estaba comprendida por todos los pacientes derivados al servicio de laboratorio entre julio y diciembre de 2017 para que se realice el examen para descarte de infección por Helicobacter pylori y que cumplían con los criterios de inclusión y exclusión.

Los criterios de inclusión fueron: Pacientes mayores de 12 años, con o sin sintomatología sugerente a infección por Helicobacter pylori posterior al examen médico y revisión de historia clínica. Los criterios de exclusión fueron: Pacientes con resultado anterior de infección por Helicobacter pylori, en tratamiento para la infección por Helicobacter pylori o pacientes operados por enfermedad gástrica.

El procedimiento de extracción de muestra sanguínea se realizó según el Manual Procedimientos de Laboratorio elaborado por el Instituto Nacional de Salud (9). Para la determinación de anticuerpos contra H. pylori, se utilizó la prueba rápida OnSite $H$. pylori Ab Combo Rapid Test para suero, plasma y sangre total de CTK Biotech que tiene una sensibilidad 93,55 $\%$, especificidad 96,94\% (10). Se siguieron los pasos establecidos en el inserto de la prueba rápida para el procedimiento e interpretación de resultados para la infección por Helicobacter pylori.

\section{Análisis de datos:}

Se utilizó el software Excel 2016 para la realización de la base datos, gráficos y tablas, así como la distribución de frecuencias y estadística descriptiva.

El estudio no fue revisado por un Comité de ética por tratarse de una intervención de vigilancia en salud pública por parte del centro de salud Ocaña

\section{RESULTADOS}

Se realizó el tamizaje a 107 personas. En el gráfico 1, se muestra la frecuencia relativa de casos de infección por $H$. pylori según mes, de julio a diciembre de 2017 , obteniéndose una frecuencia de $67,3 \%$ casos positivos. 


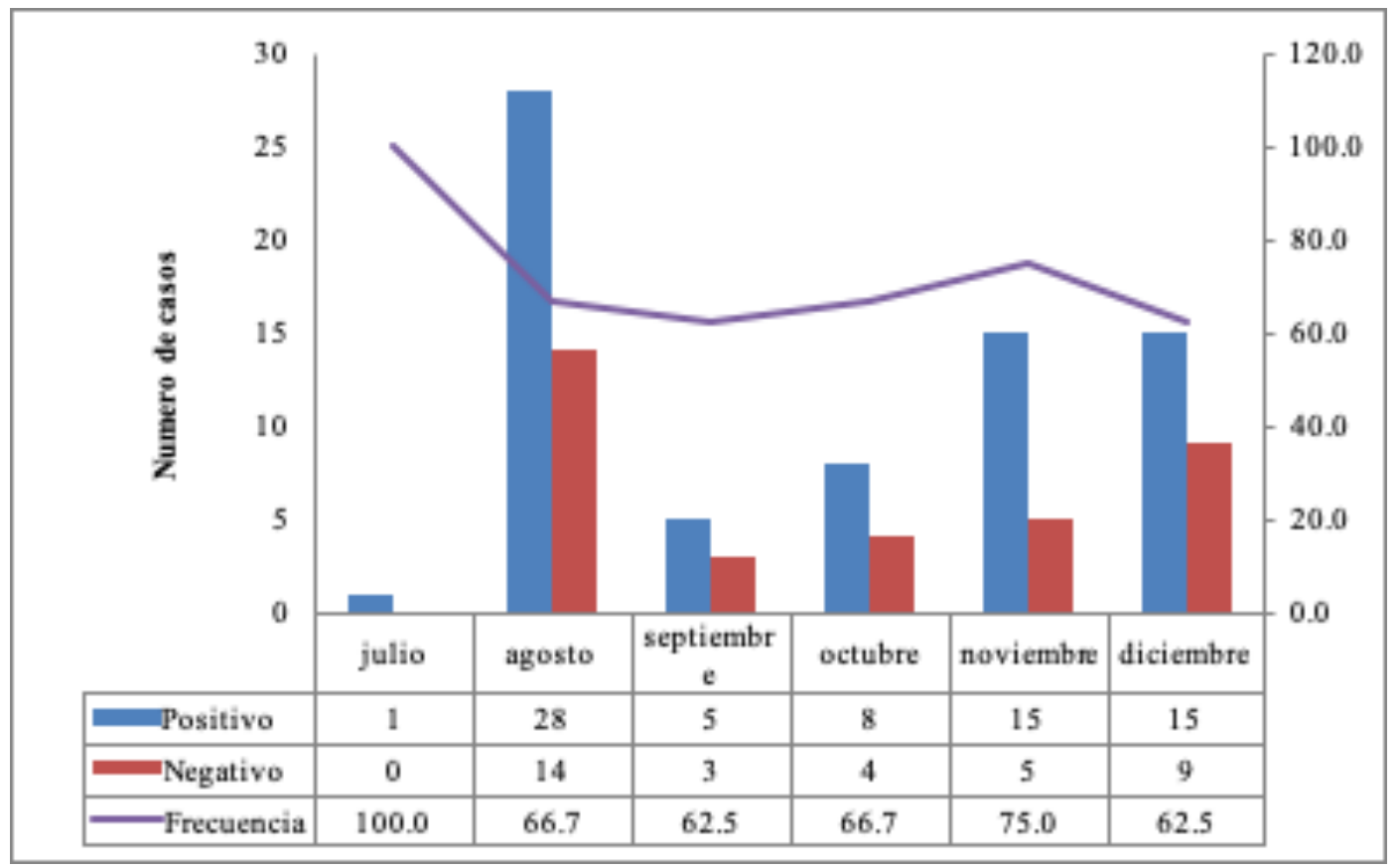

Gráfico 1. Frecuencia relativa de casos de infección por H. pylori según mes. Distrito de Ocaña, Julio a diciembre de 2017.

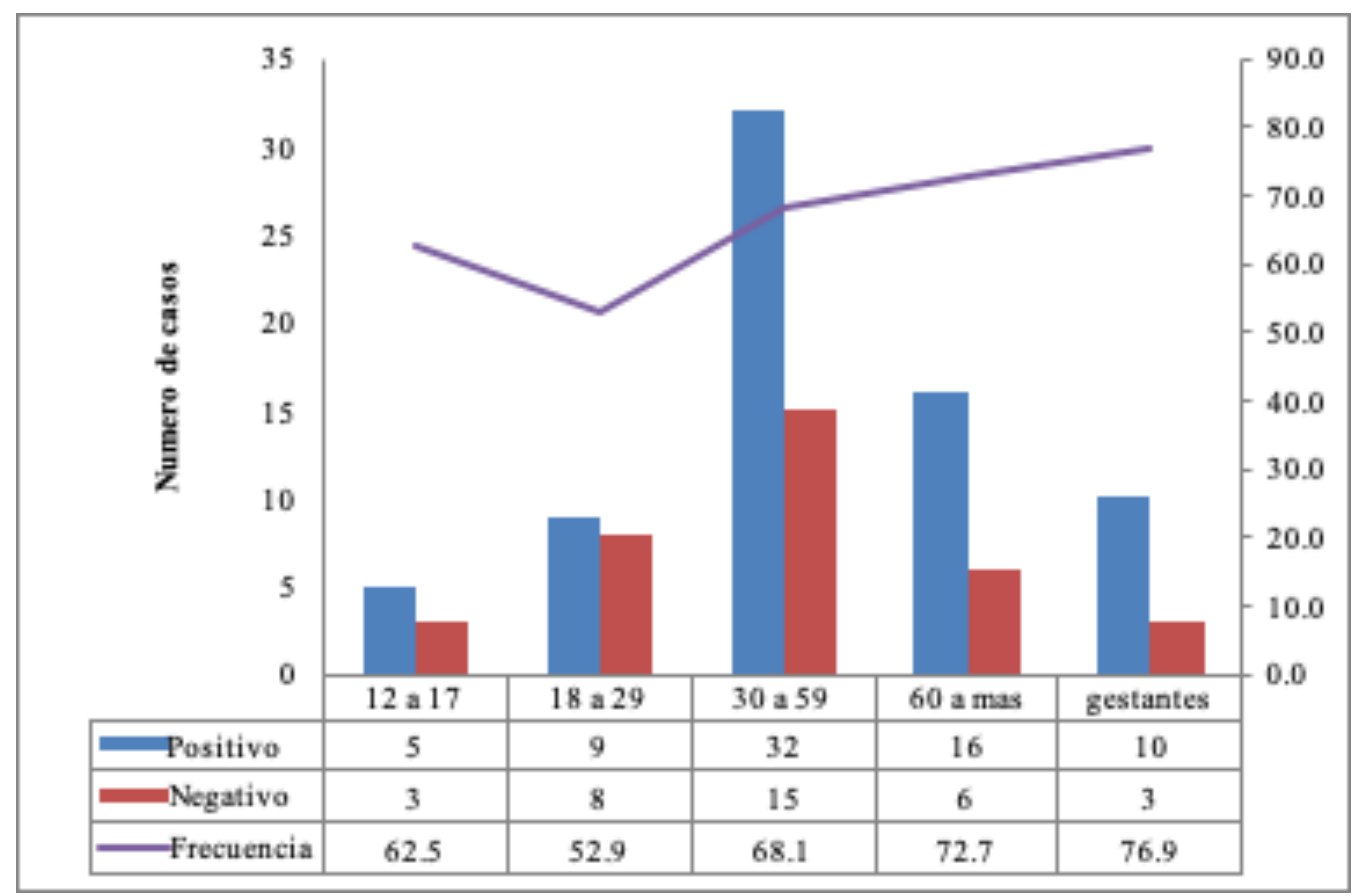

Gráfico 2. Frecuencia relativa de casos de infección por $H$. pylori según grupo etario. Distrito de Ocaña, Julio a diciembre de 2017.

En el gráfico 2, se puede observar la frecuencia relativa de casos de infección por $H$. pylori según grupo etario. Se observa una mayor frecuencia $(76,9 \%)$ en gestantes.
En la tabla 1, se muestra la frecuencia de casos de infección por $H$. pylori según sexo, siendo la frecuencia total de $69,8 \%$ para varones y $65,6 \%$ para el sexo femenino. 
Tabla 1. Frecuencia de casos de infección por Helicobacter pylori según sexo. Distrito de Ocaña, Julio a diciembre de 2017.

\begin{tabular}{|c|c|c|c|c|c|c|c|c|}
\hline \multirow{2}{*}{ Mes } & \multicolumn{4}{|c|}{ sexo masculino } & \multicolumn{4}{|c|}{ sexo femenino } \\
\hline & Positivo & Negativo & Total & $\%$ & Positivo & Negativo & Total & $\%$ \\
\hline Julio & 1 & 0 & 1 & 100 & 0 & 0 & 0 & 0,0 \\
\hline Agosto & 11 & 5 & 16 & 68,8 & 17 & 9 & 26 & 65,4 \\
\hline Septiembre & 2 & 1 & 3 & 66,7 & 3 & 2 & 5 & 60,0 \\
\hline Octubre & 3 & 1 & 4 & 75,0 & 5 & 3 & 8 & 62,5 \\
\hline Noviembre & 6 & 3 & 9 & 66,7 & 9 & 4 & 13 & 69,2 \\
\hline Diciembre & 7 & 3 & 10 & 70,0 & 8 & 4 & 12 & 66,7 \\
\hline TOTAL & 30 & 13 & 43 & 69,8 & 42 & 22 & 64 & 65,6 \\
\hline
\end{tabular}

$\mathrm{p}=0,6804$ Prueba exacta de Fisher entre hombres y mujeres.

Tabla 2. Frecuencia de casos de infección por Helicobacter pylori según comunidad. Distrito de Ocaña, Julio a diciembre de 2017.

\begin{tabular}{lrrrc}
\hline \multirow{2}{*}{ COMUNIDAD } & \multicolumn{5}{c}{ Prueba rápida OnSite H. pylori Ab Combo } \\
\cline { 2 - 5 } & Positivo & Negativo & Total & $\%$ \\
\hline Ocaña & 21 & 8 & 29 & 72,4 \\
Chuya & 4 & 2 & 6 & 66,7 \\
San José de Tomate & 14 & 6 & 20 & 70,0 \\
Sonconche & 12 & 5 & 17 & 70,6 \\
San Juan de Luren & 8 & 4 & 12 & 66,7 \\
Ocobamba & 2 & 1 & 3 & 66,7 \\
Sanquimayo & 4 & 2 & 6 & 66,7 \\
Lloquecancha & 2 & 2 & 4 & 50,0 \\
Habaspata & 2 & 2 & 4 & 50,0 \\
Pachaca & 2 & 2 & 4 & 50,0 \\
Otoccaccasa & 1 & 1 & 2 & 50,0 \\
TOTAL & 72 & 35 & 107 & 67,3 \\
\hline
\end{tabular}

En la tabla 2, se puede observar la frecuencia de casos de infección por $H$. pylori según comunidad, teniendo las comunidades de Ocaña, San José de Tomate y Sonconche, las más altas frecuencias.

\section{DISCUSIÓN}

El estudio se planteó con el objetivo de determinar la frecuencia de $H$. pylori en pacientes atendidos en el centro de salud Ocaña con o sin sintomatología digestiva sugerente a los cuales se les solicito la prueba rápida OnSite H. pylori Ab Combo.
Las cifras reportadas en países desarrollados fluctúan entre el 4 a $57 \%$, teniendo $17 \%$ de prevalencia en EEUU, en Europa varía entre el 24,7 a 56,9\% (154). En el 2010, la prevalencia mundial de la infección de $H$. pylori estuvo entre $7 \%$ y $87 \%$. La prevalencia promedio en Europa fue de 30\% (11).

Las cifras de prevalencia que se encontraron en nuestro estudio son menores que las encontradas en dos estudios realizados en el Hospital Carrión, el primero de ellos realizado entre los años 1997-1998 en el que se obtuvo $75,2 \%$ y el segundo de ellos entre el 2000 - 
2005 con un promedio de $66 \%$, teniendo en cuenta que el Hospital Carrión se encuentra en el Callao (12). Asimismo, es menor a la encontrada en un estudio realizado en la Clínica médica de la Universidad Peruana Cayetano Heredia, donde la prevalencia fue $78,69 \%$ y menor al estudio realizado en el $\mathrm{AAHH}$ de Pampas de San Juan de Lurigancho donde la prevalencia fue de $93 \%$ (13). El nivel socioeconómico se relaciona con la mayor prevalencia de la infección (14-16), esto puede explicar las diferencias de prevalencia entre ambos distritos estudiados.

Se reporta en el Perú, que la prevalencia de la infección por Helicobacter pylori ha disminuido en el curso de las últimas décadas, así como las enfermedades asociadas a ella (gastritis crónica activa, úlcera péptica y cáncer gástrico) $(17,18)$.

En el estudio de Toledo realizado en Chile se encontró que la población mayor de 60 años presentó una menor prevalencia de infección y señalan que el $34,4 \%$ de los niños son seropositivos para $H$. pylori; el $47 \%$ de la prevalencia observada en el grupo comprendido entre los 11 y los 20 años (19).

Juan Pablo Ortega et al. (20), en Chile, encontraron una prevalencia de $73 \%$ en población adulta y se asoció a gastritis de intensidad variable, generalmente asintomática. Corti R. (21), encontró en Argentina una prevalencia en adultos entre $52 \%$ y $56 \%$. Otros estudios, refieren prevalencias de $64 \%$ en la Habana (22), 69\% en Bogotá (23), 63,03\% en Ecuador (24) y $68,82 \%$ en el Hospital de Lambayeque (25); contrariamente, Lagunas y Calva en México reportan una prevalencia de $24,5 \%$ (26) y Naranjos Arcos (27), reporta una prevalencia de $24,18 \%$ en niños, en el Hospital Metropolitano de Quito.

Aliaga (28), menciona que la infección está asociada al sexo preferentemente femenino. Cifras entre el $80-90 \%$ de prevalencia cuya característica principal es que disminuye en relación al grupo etario, y predominio discreto en mujeres jóvenes (29); sin embargo, Sulca (30), encontró que no existe asociación significativa entre la infección y el sexo. En 2002 en un estudio realizado en el Policlínico Peruano-japonés de Lima, se encontró una prevalencia de infección por H. pylori de $40 \%$, sin diferencias significativas en el predominio de la infección y su relación a los factores sexo, estrato socioeconómico y tiempo de enfermedad (31). En nuestro trabajo no encontramos diferencia significativa con respecto a la infección por H. pylori y el sexo.
En el estudio de Custodio (32), la mayor parte de la población con adenocarcinoma gástrico positiva a la bacteria en Lima, proviene de 3 distritos. En el distrito de San Juan de Lurigancho se observa la mayor prevalencia de $H$. pylori con $12,7 \%$, seguido por el distrito del Callao con $9,8 \%$ y la zona rural de Lima con 7,8\%. Mientras que, los distritos que mostraron menos prevalencia fueron: La Molina $1 \%$, Miraflores 1\% y San Miguel 1\%. Pilco et al. (33), encontraron que los distritos con más alta incidencia de cáncer gástrico fueron Puente Piedra, Lince, Villa El Salvador, El Agustino, Breña y Rímac; los distritos con menos incidencia de esta enfermedad fueron San Isidro y Miraflores, observándose una relación de la prevalencia con nivel socioeconómico del distrito.

En nuestro trabajo, las comunidades presentan una frecuencia casi homogénea, por encima de 50\% de casos positivos; hay que mencionar que todas las comunidades del distrito de Ocaña están consideradas dentro del quintil de pobreza I y II.

En Lima, el último registro acerca de la relación de H. pylori y cáncer de estómago se realizó en el periodo 2010-2012 por el Ministerio de Salud, no se mencionan tasas de prevalencia para esta relación, sin embargo, se menciona a la bacteria como un factor de riesgo importante (34).

La frecuencia de infección por $H$. pylori mediante la prueba rápida $H$. pylori $\mathrm{Ab}$ Combo está por encima de países desarrollados, pero concuerda con la prevalencia reportada para Sudamérica y para países en vías de desarrollo (35).

Aunque la mayor frecuencia respecto a la cantidad de personas afectadas se da en la población adulta (3059 años) no se halla diferencias significativas con los demás grupos etarios.

Una limitación de nuestro estudio es que la prueba serológica no distingue entre infección activa o previa y no es concluyente para determinar la infección por H. pylori.

En conclusión, la frecuencia de infección por H. pylori encontrada en el estudio, es similar a la prevalencia reportada para Sudamérica y para países en vías de desarrollo. No existe diferencias significativas con respecto al sexo y al grupo etario en el distrito de Ocaña. 


\section{Agradecimientos:}

A la Unidad Ejecutora Salud Sur Ayacucho, al Centro de Ocaña, al M.C. Hector Juan Carlos Arotuma Granda y al M.C. Nestor Javier Castillon Anyosa.

\section{Declaración de financiamiento y de conflictos de intereses:}

El estudio fue financiado por el autor, declara no tener conflictos de interés.

\section{Correspondencia:}

John Christian Chávez Barriga

Coronel Cisneros S/N - Sapallanga. Huancayo,

Junín, Perú

Correo electrónico: aguilatlv@hotmail.com

Teléfono 511-990087880

\section{REFERENCIAS BIBLIOGRÁFICAS}

1. International Agency for Research on Cancer. GLOBOCAN 2008 [Internet]. Lyon: OMS; c2010 [citado el 14 de enero de 2013]. Disponible en: http:// globocan.iarc.fr/

2. Uemura N, Okamoto $\mathrm{S}$, Yamamoto $\mathrm{S}$, et al. Helicobacter pylori infection and the development of gastric cancer. N Engl J Med. 2001; 345:784-9.

3. Sachs G, Scott DR. Helicobacter pylori: Eradication or Preservation. F1000 Med Rep. 2012; 4:7.

4. Go MF. Natural history and epidemiology of Helicobacter pylori infection article. Aliment Pharmacol Ther. 2002; 16:3-15.

5. IARC Working Group on the Evaluation of Carcinogenic Risks to Humans. Schistosomes, liver flukes and Helicobacter pylori. IARC Monogr Carcinog Risks Hum.1994; 61:1-241.

6. Fernández-Delgado $\mathrm{M}$, Contreras $\mathrm{M}$, GarcíaAmado MA, Michelangeli F, Suárez P. Evidencias de la transmisión acuática de Helicobacter pylori. INCI [Revista en internet] Interciencia., 2008; 33(6): 412-7. (citado el enero de 2020). Disponible en: https://www.redalyc.org/articulo.oa?id=33933603

7. Hultén K, Enroth H, Nyström T, Engstrand L. Presence of Helicobacter species DNA in Swedish water. Appl Microbiol. 1998; 85(2):282-6.

8. Montero-Campos V, Hernández-Soto A, MasísMeléndez F, et al. Hallazgo de la bacteria Helicobacter pylori en agua de consumo humano y su relación con la incidencia de cáncer gástrico en Costa Rica. Tecnología en Marcha. 2011; 24(3):3-14.

9. Zurita S. Procedimientos de Laboratorio: Manual: Laboratorios locales I, Laboratorios locales II. Lima, Perú: Ministerio de Salud; Instituto Nacional de Salud; 2013.p. 554.
10. Chey WD, Wong BC. American College of Gastroenterology guideline on the management of Helicobacter pylori infection. Am J Gastroenterol 2007; 102:1808-25.

11. Rupnow MF, Chang AH, Shachter RD. Costeffectiveness of a potential prophylactic Helicobacter pylori vaccine in the United States. J Infect Dis. 2009; 200:1311-17.

12. Bevilacqua R. Prevalencia de la infección por Helicobacter pylori en pacientes sintomáticos atendidos en consulta externa en el Hospital I Octavio Mongrut Muñoz-RAS-EsSalud periodo 2007-2010. Tesis para título de Especialidad. Lima, Perú: Universidad Nacional Mayor de San Marcos; 2014. $45 \mathrm{pp}$.

13. Prochazka R, Salazar F, Barriga E, Salazar F. Prevalencia de Helicobacter pylori en una Clínica Privada de Lima. Sensibilidad de las Biopsias del Antro y el Cuerpo, y la Prueba Rápida de la Ureasa. Rev Gastroenterol Perú. 2010; 30-1: 33-39.

14. Macenlle R. Prevalencia de la infección por Helicobacter pylori en la población general adulta de la provincia de Ourense y estudio de factores de riesgo asociados. Universidad de Santiago de Compostela. Memoria presentada para optar al grado de Doctor en Medicina. España 2007.

15. Go MF. Review article: natural history and epidemiology of Helicobacter pylori infection. Aliment Pharmacol Ther. 2002; 16(suppl 1):3-15.

16. Yamada T, Alpers DH, Laine L. Stomach. In: Yamada's textbook of gastroenterology. $4^{\circ}$ ed. USA: Lippincott Williams \& Wilkins Publishers; 2003. 1: p. $482-488$

17. Mendoza D, Herrera P, Gilman RH, et al. Variation in the prevalence of gastric cancer in Perú. Int $\mathrm{J}$ Cancer. 2008; 123(2):414-20.

18. Ramírez-Ramos A, Watanabe-Yamamoto J, TakanoMorón J. Decrease in prevalence of peptic ulcer and gastric adenocarcinoma at the Policlínico Peruano Japonés, Lima, Perú, between the years 1985 and 2002. Analysis of 31446 patients. Acta Gastroenterol LatinoAm. 2006; 36(3):66-73.

19. Toledo H, Defilippi C, Madrid AM., et al. Prevalencia de la infección por Helicobacter pylori según ensayo de la ureasa en pacientes derivados a la Unidad de Endoscopía del Hospital Clínico de la Universidad de Chile. Rev Hosp Clin Univ Chile. 2007; 18:189-93.

20. Ortega JP, Espino A, Calvo A, et al. Infección por Helicobacter pylori en pacientes sintomáticos con patología gastroduodenal benigna. Análisis de 5664 pacientes. Rev Med Chile. 2010; 138:529-535.

21. Corti RE. Helicobacter pylori: algunos aspectos epidemiológicos en Latinoamérica luego de un cuarto de siglo. Acta Gastroenterol LatinoAm. 2009; 39:175176. 
22. Ramírez A, Mendoza E, Leey J, Guerrera J. Estudio de Helicobacter pylori en el Perú. Rev Peru Med Exp Salud Pública. 2004; 19(4):209-14.

23. Klein P, Gilman R, Leon-Barua R, Díaz F, O’brian S and Graham D. The epidemiology of Helicobacter pylori in Peruvian children between 6 and 30 Months of Age. Am j Gastroenterol. 1994; 89(12):2196-2200.

24. Pon R, Torres M, Chanis R. Estudio descriptivo Ç de la infección por Helicobacter pylori en pacientes pediátricos del Hospital del Niño entre junio y diciembre del 2002. Hosp Niño Panamá. 2004; 20(2):124-127.

25. Lagunas B, Calva R, Ramírez E. Prevalencia de Helicobacter pylori en niños sanos en edad escolar. Rev Mex Patol Clin. 2001; 48(1):23-26.

26. Naranjos Arcos M. Prevalencia de enfermedades gastrointestinales diagnosticadas por endoscopías en niños atendidos en el Hospital Metropolitano de Quito 2010. Disponible en: http://repositorio.usfq. edu.ec/bitstream/23000/376/1/97840.pdf

27. Jara-Romero L, Sánchez-Figueroa C, Santana-Bazalar D, León-Jiménez F, Cubas-Benavides F. Frecuencia de Helicobacter pylori y características clínicas en niños con endoscopía digestiva alta de un hospital de Lambayeque: 2007 - 2010. Rev Cuerpo Méd HNAAA. 2013; 6(3):28-32.

28. Aliaga J. Prevalencia de Helicobacter Pylori en pacientes con sintomatología digestivo alta de la población de Ayacucho. Tesis para obtener el Título de Biólogo. Ayacucho, Perú: Universidad Nacional de San Cristóbal de Huamanga; 1998. 67 pp.

29. The Gastrointestinal Physiology Working Group. Helicobacter pylori and Gastritis in Peruvian patients relationship to socioeconomic level, age and sex. Am J Gastroent. 1990; 86:819-823.
30. Sulca S. Prevalencia de Helicobacter pylori en pacientes con sintomatología gástrica. Hospital Tipo II EsSalud Huamanga. Agosto - Diciembre, 2007. Tesis para obtener el título de Biólogo. Ayacucho, Perú: Universidad Nacional de San Cristóbal de Huamanga; 2007. 75 pp.

31. Ruiz P. Prevalencia de la Infección por Helicobacter pylori en el Policlínico Peruano Japonés. Tesis para optar el título profesional de especialista en Gastroenterología Lima, Perú. Universidad Nacional Mayor de San Marcos; 2002. 92 pp.

32. Custodio D. Prevalencia de la infección de la bacteria Helicobacter pylori en pacientes diagnosticados con adenocarcinoma gástrico en el Instituto Nacional de Enfermedades Neoplásicas (INEN). Tesis para optar el Título Profesional de Licenciado en Biología. Lima, Perú: Universidad Ricardo Palma; 2017. 109 pp.

33. Pilco P, Payet E, Cáceres E. Cáncer Gástrico en Lima Metropolitana. Rev Gastroenterol. Perú. 2006; 26:377-385.

34. Instituto Nacional de Enfermedades Neoplásicas. Registro de cáncer de lima metropolitana incidencia y mortalidad 2010 - 2012. Lima, Perú: Ministerio de Salud; 2016. 173 pp.

35. Passaro D, Taylor D, Meza R, Cabrera L, Gilman R and Parsonnet J. Acute Helicobacter pylori Infection Is Followed by an Increase in Diarrheal Disease Among Peruvian Children. Pediatrics. 2000; 108(5):87-95

Recibido: 21/02/2018

Aceptado: 28/01/2020 